\title{
Zero sets of holomorphic functions in the Nevanlinna type class on convex domains in $\mathbb{C}^{2}$
}

\author{
By Heungju AhN and Hong Rae CHо \\ (Received May 21, 2001) \\ (from Nagoya Mathematical Journal)
}

\begin{abstract}
We study a problem concerning zero sets of holomorphic functions in the Nevanlinna type class on convex domains (not necessarily finite type) in $\mathbb{C}^{2}$. We give a Blaschke type condition for the zero set defined by a function in the Nevanlinna type class on convex domains in $\mathbb{C}^{2}$. Moreover, we present an example of a convex domain of exponentially-flat infinite type on which it is possible to give sufficient conditions for zero sets of holomorphic functions in the classical Nevanlinna class.
\end{abstract}

\section{Introduction and statement of the results}

In this paper, we give a Blaschke type condition for the zero set of holomorphic functions in the Nevanlinna type class on convex domains in $\mathbb{C}^{2}$ without the finite type condition of domains.

Let $D=\{\rho<0\}$ be a bounded domain in $\mathbb{C}^{n}, \rho \in C^{2}\left(\mathbb{C}^{n}\right)$ and $d \rho \neq 0$ on $\partial D$. For $\alpha>0$ the Nevanlinna class of order $\alpha$ denoted by $\mathcal{N}_{\alpha}(D)$ consists of the holomorphic functions $f$ in $D$ satisfying

$$
\int_{D}|\rho(z)|^{\alpha-1}|\log | f(z)|| d V(z)<\infty
$$

In particular, let $\mathcal{N}(D)=\mathcal{N}_{0}(D)$ be the Nevanlinna class consisting of the holomorphic functions $f$ in $D$ satisfying

$$
\sup _{\epsilon>0} \int_{\partial D_{\epsilon}}|\log | f(z)|| d \sigma_{\epsilon}(z)<\infty
$$

2000 Mathematics Subject Classification. 32A22, 32 A26.

Key words and Phrases. Convex domains, Poincaré-Lelong equation, Blaschke condition of order $\alpha$, Nevanlinna class of order $\alpha$, weighted $L^{1}$-estimates for the $\bar{\partial}$-equation.

The first author was partly supported by GARC and the second author was supported by grant No. R01-2000-000-00001-0 from the Basic Research Program of the Korea Science \& Engineering Foundation. 
Here $\partial D_{\epsilon}=\{\rho=-\epsilon\}$ and $d \sigma_{\epsilon}$ denotes the Lebesgue measure on $\partial D_{\epsilon}$. Let $X$ be a zero set of a holomorphic function on $D$. Let $X_{k}$ be the irreducible components of $X$ and $n_{k}$ the corresponding multiplicities of $X_{k}$. Classically the data $X=\sum_{k} n_{k} X_{k}$ is called a divisor. For strictly pseudoconvex domains with some topological condition, in the mid 1970's, Henkin [Hen], Skoda [Sko] and Dautov-Henkin [DaHe] proved that a divisor $X$ satisfies the Blaschke condition of order $\alpha$,

$$
\sum_{k} n_{k} \int_{X_{k}}|\rho(z)|^{\alpha+1} d \sigma_{X_{k}}(z)<\infty
$$

if and only if $X$ is the zero set of a function of class $\mathcal{N}_{\alpha}(D), \alpha \geq 0$, where $d \sigma_{X_{k}}$ denotes the Lebesgue measure on the regular part of $X_{k}$. Bonami-Charpentier $[\mathrm{BoCh}]$ proved the same results on complex ellipsoids in 1982 . In 1988 , by using estimates for the $\bar{\partial}$-Neumann problem on pseudoconvex domains in $\mathbb{C}^{2}$ of finite type, Chang-Nagel-Stein [CNS] proved that the Blaschke condition is sufficient for the zero set of a function in the Nevanlinna class. Bruna-Charpentier-Dupain [BCD] proved the same result as Chang-Nagel-Stein's for the case of smoothly bounded convex domains of finite type in $\mathbb{C}^{n}$ which are strict type. Without the condition of finite type for convex domains, if we strengthen the Blaschke type condition we can prove the following theorem.

Theorem 1.1. Let $D=\{\rho<0\}$ be a bounded convex domain in $\mathbb{C}^{2}, \rho \in$ $C^{2}\left(\mathbb{C}^{2}\right), \partial D=\{\rho=0\}$ and $d \rho \neq 0$ on $\partial D$. Let $\alpha>0$. Assume that a divisor $X$ in $D$ satisfies the Blaschke condition of order $\alpha-1 / 2$, i.e.,

$$
\sum_{k} n_{k} \int_{X_{k}}|\rho(z)|^{\alpha+1 / 2} d \sigma_{X_{k}}<\infty
$$

Then there is a function $h \in \mathcal{N}_{\alpha}(D)$ satisfying $X=\{z \in D ; h(z)=0\}$.

Recently Cumenge [Cum] and Diederich-Mazzilli [DiMa] obtained independently the characterization of the zero sets of functions in the Nevanlinna class of order $\alpha \geq 0$, in smoothly bounded convex domains of finite type in $\mathbb{C}^{n}$ without the strict type condition.

For $s>0$, define

$$
D_{s}=\left\{\left(z_{1}, z_{2}\right) \in \mathbb{C}^{2} ;\left|z_{1}\right|^{2}+c \cdot \exp \left(-\frac{1}{\left|z_{2}\right|^{s}}\right)<1\right\},
$$

where $c=\exp (1+2 / s)$. Then $D_{s}$ is a bounded convex domain of $C^{\infty}$ class and any point of the form $\left(e^{i \theta}, 0\right)$ in the boundary of $D_{s}$ is a typical example of a nonLevi flat infinite type boundary point. Except these boundary points all boundary points are strongly pseudoconvex. 
The proof of Theorem 1.1 depends essentially on weighted $L^{1}(D)$-estimates for $\bar{\partial}$. For the case of $\alpha=0$ in Theorem 1.1 we need the $L^{1}(\partial D)$-estimate for $\bar{\partial}$. However, we cannot prove the $L^{1}(\partial D)$-estimate for $\bar{\partial}$ on convex domains without finite type condition. Quite surprisingly, we discovered that even though $D_{s}$ is exponentially-flat infinite type, the $L^{1}(\partial D)$-estimate for $\bar{\partial}$ depends on the grade of flatness of $D_{s}$. If $0<s<1$, we prove the $L^{1}(\partial D)$-estimate for $\bar{\partial}$ on $D_{s}$ in Theorem 4.1. Thus we obtain the following result.

Theorem 1.2. Let $0<s<1$. Let $X$ be a divisor in $D_{s}$ that satisfies the Blaschke condition of order $-1 / 2$. Then $X$ is defined by a function in $\mathcal{N}\left(D_{s}\right)$.

REMARK 1.3. In Theorems 1.1 and 1.2 our Blaschke type conditions are only sufficient ones. It is well-known that for every smoothly bounded domain $D$ the divisor $X$ of any function $\mathcal{N}_{\alpha}(D), \alpha \geq 0$, satisfies the Blaschke condition of order $\alpha([\mathrm{DaHe}],[\mathrm{Sko}])$.

\section{Weighted $L^{1}$-estimate for the $\bar{\partial}$-equation}

Let $D=\{\rho<0\}$ be a bounded convex domain in $\mathbb{C}^{2}, \rho \in C^{2}\left(\mathbb{C}^{2}\right), \partial D=\{\rho=$ $0\}$ and $d \rho \neq 0$ on $\partial D$. In this section we prove the following weighted $L^{1}$-estimates for $\bar{\partial}$ which are essential for proving Theorem 1.1. In the case $p>1, L^{p}$ estimates for $\bar{\partial}$ was proved by Polking [Pol]. The $L^{1}(D)$-estimate for $\bar{\partial}$ on convex domains in $\mathbb{C}^{2}$ was one of the problems presented by Range (see Problem 4 of $\S 6$ in [Ra3]).

Theorem 2.1. Let $\alpha>0$. Then there is a solution $u$ to $\bar{\partial} u=f$ in $D$ such that

$$
\int_{D}|\rho(z)|^{\alpha-1}|u(z)| d V(z) \leq C_{\alpha} \int_{D}|\rho(z)|^{\alpha}\left(|f(z)|+\frac{|f(z) \wedge \bar{\partial} \rho(z)|}{|\rho(z)|}\right) d V(z)
$$

provided that the right-hand side is finite for $(0,1)$-form $f$ in $D$ with $\bar{\partial} f=0$. Here $C_{\alpha}$ is a constant depending only on $\alpha$.

One of the crucial points for proving Theorem 2.1 is to construct a certain weighted solution formula. We define

$$
\tilde{\phi}(\zeta, z)=\sum_{j=1}^{2} \frac{\partial \rho}{\partial \zeta_{j}}(\zeta)\left(\zeta_{j}-z_{j}\right)-\rho(\zeta)
$$

The following is a well-known consequence of the convexity of $D$ :

$$
2 \operatorname{Re} \tilde{\phi}(\zeta, z) \geq-\rho(\zeta)-\rho(z) \text { for all } \zeta, z \in \bar{D}
$$


For any $r>1$ we can define a kernel

$$
\begin{aligned}
K^{r}(\zeta, z)= & c_{0, r} \frac{\rho(\zeta)^{r}}{|\zeta-z|^{4} \tilde{\phi}(\zeta, z)^{r}} \partial_{\zeta}|\zeta-z|^{2} \wedge \bar{\partial}_{\zeta} \partial_{\zeta}^{\prime}|\zeta-z|^{2} \\
& +c_{1, r} \frac{\rho(\zeta)^{r+1}}{|\zeta-z|^{2} \tilde{\phi}(\zeta, z)^{r+1}} \partial_{\zeta}|\zeta-z|^{2} \wedge \partial \bar{\partial} \log \frac{1}{-\rho(\zeta)}
\end{aligned}
$$

which induces a solution operator

$$
S f(z)=\int_{\zeta \in D} f(\zeta) \wedge K^{r}(\zeta, z), \quad z \in D
$$

such that

$$
f=\bar{\partial}(S f)
$$

for a continuous $(0,1)$-form $f$ in $\bar{D}$ with $\bar{\partial} f=0$ (see [BeAn]). For a smooth form $f$, this formula holds for any $r>0$. Note that

$$
\partial \bar{\partial} \log \frac{1}{-\rho}=\frac{\partial \rho \wedge \bar{\partial} \rho}{\rho^{2}}-\frac{\partial \bar{\partial} \rho}{\rho} .
$$

Thus

$$
K^{r}(\zeta, z)=|\rho(\zeta)|^{r} K_{1}^{r}(\zeta, z)+\bar{\partial} \rho(\zeta) \wedge|\rho(\zeta)|^{r-1} K_{2}^{r}(\zeta, z)+|\rho(\zeta)|^{r} K_{3}^{r}(\zeta, z)
$$

where

$$
\left|K_{1}^{r}(\zeta, z)\right| \lesssim \frac{1}{|\zeta-z|^{3}|\tilde{\phi}(\zeta, z)|^{r}}
$$

and

$$
\left|K_{j}^{r}(\zeta, z)\right| \lesssim \frac{1}{|\zeta-z||\tilde{\phi}(\zeta, z)|^{r+1}}
$$

for $j=2,3$.

Lemma $2.2([\mathrm{Pol}])$. Let $\left(\zeta_{0}, z_{0}\right) \in \partial D \times \partial D$ such that $\tilde{\phi}\left(\zeta_{0}, z_{0}\right)=0$. Then there exist neighborhoods $V$ of $z_{0}$ and $W$ of $\zeta_{0}$ such that for each $\zeta \in W$, there exists a $C^{1}$ local coordinate system $z \mapsto t^{(\zeta)}(z)=\left(t_{1}, t_{2}, t_{3}, t_{4}\right)$ on $V$ with the following properties:

$$
\begin{gathered}
t_{1}(z)=\rho(\zeta)-\rho(z), \quad t_{2}(z)=\operatorname{Im} \tilde{\phi}(\zeta, z), \quad t_{3}(\zeta)=t_{4}(\zeta)=0 \\
\left|t^{(\zeta)}(z)-t^{(\zeta)}\left(z^{\prime}\right)\right| \sim\left|z-z^{\prime}\right|
\end{gathered}
$$

for all $z, z^{\prime} \in V$ with the constants in (2.3) independent of $\zeta \in W$. 
LEMMA 2.3. Let $r$ be sufficiently large. If $\alpha>0$ and $\alpha-\epsilon>-1$, then there exists a constant $C_{\alpha, \epsilon}$ such that for $j=1,2$

$$
\int_{z \in D}|\rho(z)|^{\alpha-\epsilon}\left|K_{j}^{r}(\zeta, z)\right| d V(z) \leq C_{\alpha, \epsilon}|\rho(\zeta)|^{\alpha-\epsilon-r+1} \quad \text { for } \quad \zeta \in D .
$$

Proof. We fix $\left(\zeta_{0}, z_{0}\right) \in \partial D \times \partial D$ such that $\tilde{\phi}\left(\zeta_{0}, z_{0}\right)=0$ and use the coordinate system $t=\left(t_{1}, t_{2}, t_{3}, t_{4}\right)$ of $(2.2)$. It is enough to prove the inequality

$$
\int_{z \in D \cap V}|\rho(z)|^{\alpha-\epsilon}\left|K_{j}^{r}(\zeta, z)\right| d V(z) \lesssim|\rho(\zeta)|^{\alpha-\epsilon-r+1} \quad \text { for } \quad \zeta \in D \cap W
$$

Put $w=t_{3}+i t_{4}$. For the case $j=1$ it follows from (2.1) that

$$
\begin{aligned}
J_{1}(\zeta) & =\int_{z \in D \cap V}|\rho(\zeta)|^{\alpha-\epsilon}\left|K_{1}^{r}(\zeta, z)\right| d V(z) \\
& \lesssim \int_{\substack{w \in \mathbb{C} \\
|w|<R}} \int_{-T_{2}}^{T_{2}} \int_{0}^{T_{1}} \frac{t_{1}^{\alpha-\epsilon} d w d t_{1} d t_{2}}{\left(|w|+t_{1}+\left|t_{2}\right|\right)^{3}\left(t_{1}+\left|t_{2}\right|+|\rho(\zeta)|\right)^{r}} \\
& \lesssim \int_{-T_{2}}^{T_{2}} \int_{0}^{T_{1}} \frac{t_{1}^{\alpha-\epsilon} d t_{1} d t_{2}}{\left(t_{1}+\left|t_{2}\right|\right)\left(t_{1}+\left|t_{2}\right|+|\rho(\zeta)|\right)^{r}}
\end{aligned}
$$

We make the change of variables $t_{1}=|\rho| t_{1}^{\prime}$ and $t_{2}=|\rho| t_{2}^{\prime}$, and omit the primes, this becomes

$$
J_{1}(\zeta) \lesssim|\rho(\zeta)|^{\alpha-\epsilon-r+1} \int_{\left(t_{1}, t_{2}\right) \in \mathbb{R}^{2}} \frac{\left|t_{1}\right|^{\alpha-\epsilon} d t_{1} d t_{2}}{\left(\left|t_{1}\right|+\left|t_{2}\right|\right)\left(\left|t_{1}\right|+\left|t_{2}\right|+1\right)^{r}}
$$

If $\alpha-\epsilon \geq 0$, choose $r$ such that $r-\alpha+\epsilon>1$. We have

$$
\begin{aligned}
J_{1}^{\prime} & =\int_{\left(t_{1}, t_{2}\right) \in \mathbb{R}^{2}} \frac{\left|t_{1}\right|^{\alpha-\epsilon} d t_{1} d t_{2}}{\left(\left|t_{1}\right|+\left|t_{2}\right|\right)\left(\left|t_{1}\right|+\left|t_{2}\right|+1\right)^{r}} \\
& \lesssim \int_{0}^{\infty} \frac{d \tau}{(\tau+1)^{r-\alpha+\epsilon}} \lesssim 1
\end{aligned}
$$

Now if $-1<\alpha-\epsilon<0$, then

$$
\begin{aligned}
J_{1}^{\prime} & =\int_{\left(t_{1}, t_{2}\right) \in \mathbb{R}^{2}} \frac{d t_{1} d t_{2}}{\left|t_{1}\right|^{\epsilon-\alpha}\left(\left|t_{1}\right|+\left|t_{2}\right|\right)\left(\left|t_{1}\right|+\left|t_{2}\right|+1\right)^{r}} \\
& \lesssim \int_{0}^{\infty} \int_{0}^{\infty} \frac{d t_{1} d t_{2}}{\left|t_{1}\right|^{\epsilon-\alpha+\eta}\left|t_{2}\right|^{1-\eta}\left(\left|t_{1}\right|+\left|t_{2}\right|+1\right)^{r}} \\
& \lesssim \int_{0}^{\infty} \frac{d t_{1}}{\left|t_{1}\right|^{\epsilon-\alpha+\eta}\left(\left|t_{1}\right|+1\right)^{r / 2}} \int_{0}^{\infty} \frac{d t_{2}}{\left|t_{2}\right|^{1-\eta}\left(\left|t_{2}\right|+1\right)^{r / 2}}
\end{aligned}
$$


If we choose sufficiently small $\eta$ and large $r$ so that $0<\epsilon-\alpha+\eta<1$ and $r>2$, then we have $J_{1}^{\prime} \lesssim 1$.

For the case $j=2$ we have

$$
\begin{aligned}
J_{2}(z) & =\int_{z \in D \cap V}|\rho(z)|^{\alpha-\epsilon}\left|K_{2}^{r}(\zeta, z)\right| \\
& \lesssim \int_{z \in D \cap V}|\rho(z)|^{\alpha-\epsilon} \frac{1}{|\zeta-z||\tilde{\phi}(\zeta, z)|^{r+1}} d V(z) \\
& \lesssim \int_{\mid w \in \mathbb{C}} \int_{-T_{2}}^{T_{2}} \int_{0}^{T_{1}} t_{1}^{\alpha-\epsilon} \frac{d t_{1} d t_{2} d w}{|w|\left(t_{1}+\left|t_{2}\right|+|\rho(\zeta)|\right)^{r+1}} \\
& \lesssim \int_{-T_{2}}^{T_{2}} \int_{0}^{T_{1}} t_{1}^{\alpha-\epsilon} \frac{d t_{1} d t_{2}}{\left(t_{1}+\left|t_{2}\right|+|\rho(\zeta)|\right)^{r+1}} \\
& \lesssim|\rho(\zeta)|^{\alpha-r+1-\epsilon} \int_{-\infty}^{\infty} \int_{0}^{\infty} t_{1}^{\alpha-\epsilon} \frac{d t_{1} d t_{2}}{\left(t_{1}+\left|t_{2}\right|+1\right)^{r+1}}
\end{aligned}
$$

Now the integral

$$
\int_{-\infty}^{\infty} \int_{0}^{\infty} t_{1}^{\alpha-\epsilon} \frac{d t_{1} d t_{2}}{\left(t_{1}+\left|t_{2}\right|+1\right)^{r+1}}
$$

converges, if $\alpha-\epsilon>-1$ and $r-\alpha+\epsilon>1$. Thus we have the result.

Now we can prove Theorem 2.1. For $j=1,3$ we define

$$
I_{j}^{r} f(z)=\int_{\zeta \in D} f(\zeta) \wedge|\rho(\zeta)|^{r} K_{j}^{r}(\zeta, z)
$$

and

$$
I_{2}^{r} f(z)=\int_{\zeta \in D} f(\zeta) \wedge \bar{\partial} \rho(\zeta) \wedge|\rho(\zeta)|^{r-1} K_{2}^{r}(\zeta, z)
$$

Then Theorem 2.1 is a direct consequence of the next proposition.

Proposition 2.4. Let $\alpha>0$. Then there is a constant $C_{\alpha}$ such that for $j=1,2,3$

$$
\int_{D}|\rho(z)|^{\alpha-1}\left|I_{j}^{r} f(z)\right| d V(z) \leq C_{\alpha} \int_{D}|\rho(z)|^{\alpha}\left(|f(z)|+\frac{|f(z) \wedge \bar{\partial} \rho(z)|}{|\rho(z)|}\right) d V(z) .
$$

Proof. For the case $j=1$ it follows from Lemma 2.3 that

$$
\begin{aligned}
\int_{z \in D} & |\rho(z)|^{\alpha-1}\left|I_{1}^{r} f(z)\right| d V(z) \\
& \lesssim \int_{\zeta \in D}|f(\zeta)||\rho(\zeta)|^{r} \int_{z \in D}|\rho(z)|^{\alpha-1}\left|K_{1}^{r}(\zeta, z)\right| d V(z)
\end{aligned}
$$




$$
\lesssim \int_{\zeta \in D}|f(\zeta) \| \rho(\zeta)|^{\alpha} d V(\zeta)
$$

In the case $j=2$, we have

$$
\begin{aligned}
\int_{z \in D}|\rho(z)|^{\alpha-1}\left|I_{2}^{r} f(z)\right| d V(z) \\
\quad \lesssim \int_{\zeta \in D}|f(\zeta) \wedge \bar{\rho}(\zeta)||\rho(\zeta)|^{r-1} \int_{z \in D}|\rho(z)|^{\alpha-1}\left|K_{2}^{r}(\zeta, z)\right| d V(z) \\
\quad \lesssim \int_{\zeta \in D}|f(\zeta) \wedge \bar{\rho}(\zeta)||\rho(\zeta)|^{\alpha-1} d V(\zeta) .
\end{aligned}
$$

The case $j=3$ easily can be reduced to the case $j=2$.

\section{Poincaré-Lelong equation and zero sets for the Nevanlinna class of order $\alpha>0$}

In this section we prove Theorem 1.1. Let $X$ be a divisor of $D$. Lelong showed that $X$ is represented by a $d$-closed, positive $(1,1)$-current $\Theta_{X}$ ([Le1], [Le2]). We assume that $X$ satisfies the Blaschke condition of order $\alpha-1 / 2, \alpha>0$. Let $t_{\Theta_{X}}$ be the trace measure defined by $t_{\Theta_{X}}=\Theta_{X} \wedge \beta$, where $\beta=i \partial \bar{\partial}|z|^{2}$. Then the Blaschke condition of order $\alpha-1 / 2$ can be written as

$$
\int_{D}|\rho|^{\alpha+1 / 2} t_{\Theta_{X}}=: B\left(\Theta_{X}, \alpha-\frac{1}{2}\right)<+\infty
$$

The proof of Theorem 1.1 is reduced to finding real valued solutions of the so-called Poincaré-Lelong equation $i \partial \bar{\partial} v=\Theta_{X}$ with control of the growth of $v$, since any solution is $v=\log |g|$ for a holomorphic function $g$ that defines $X$. In fact, to prove Theorem 1.1 it suffices to show that for a $d$-closed, positive $(1,1)$-current $\Theta$ in $D$ satisfying the Blaschke condition of order $\alpha-1 / 2$, there is a real valued solution $v$ to $i \partial \bar{\partial} v=\Theta$ such that

$$
\int_{D}|\rho|^{\alpha-1}|v| d V \lesssim B\left(\Theta, \alpha-\frac{1}{2}\right)
$$

We will prove this result in Theorem 3.3. By a standard regularization and approximation argument we may assume that $\Theta$ has smooth coefficients in $\bar{D}$. First we show that the $L^{1}$-norms of $|\rho|^{\alpha-1 / 2}|\Theta \wedge \partial \rho \wedge \bar{\partial} \rho|$ and $|\rho|^{\alpha}\{|\Theta \wedge \partial \rho|+|\Theta \wedge \bar{\partial} \rho|\}$ are controlled by $B(\Theta, \alpha-1 / 2)$.

Theorem 3.1. Let $\Theta$ be a d-closed, positive smooth $(1,1)$-form satisfying the Blaschke condition of order $\alpha-1 / 2$. Let $\alpha \geq 0$. Then the following assertions are true. 
(i)

$$
\int_{D}|\rho(z)|^{\alpha-1 / 2}|\Theta(z) \wedge \partial \rho(z) \wedge \bar{\partial} \rho(z)| d V \lesssim B\left(\Theta, \alpha-\frac{1}{2}\right)
$$

(ii)

$$
\int_{D}|\rho(z)|^{\alpha}(|\Theta(z) \wedge \partial \rho(z)|+|\Theta(z) \wedge \bar{\partial} \rho(z)|) d V \lesssim B\left(\Theta, \alpha-\frac{1}{2}\right)
$$

Proof. The results for the case $\alpha>1 / 2$ where stated in [DaHe].

(i) Since $|\rho|^{\alpha-1 / 2} \Theta \wedge \partial \rho \wedge \bar{\partial} \rho / i$ is positive, it is sufficient to verify that

$$
\left.\left|\int_{D}\right| \rho\right|^{\alpha-1 / 2} \Theta \wedge \partial \rho \wedge \bar{\partial} \rho \mid \lesssim B\left(\Theta, \alpha-\frac{1}{2}\right) .
$$

By Stokes' theorem, it follows that

$$
\int_{D} d\left(|\rho|^{\alpha+1 / 2} \Theta \wedge d^{c} \rho\right)=\int_{\partial D}|\rho|^{\alpha+1 / 2} \Theta \wedge d^{c} \rho=0
$$

Since $\Theta$ is $d$-closed, we have

$$
d\left(|\rho|^{\alpha+1 / 2} \Theta \wedge d^{c} \rho\right)=-\left(\alpha+\frac{1}{2}\right)|\rho|^{\alpha-1 / 2} \Theta \wedge d \rho \wedge d^{c} \rho+|\rho|^{\alpha+1 / 2} \Theta \wedge d d^{c} \rho .
$$

Hence we have

$$
\left(\alpha+\frac{1}{2}\right) \int_{D}|\rho|^{\alpha-1 / 2} \Theta \wedge d \rho \wedge d^{c} \rho=\int_{D}|\rho|^{\alpha+1 / 2} \Theta \wedge d d^{c} \rho
$$

and

$$
\begin{aligned}
\int_{D}|\rho|^{\alpha-1 / 2} \Theta \wedge \partial \rho \wedge \bar{\partial} \rho & \sim \int_{D}|\rho|^{\alpha-1 / 2} \Theta \wedge d \rho \wedge d^{c} \rho \\
& \sim \int_{D}|\rho|^{\alpha+1 / 2} \Theta \wedge d d^{c} \rho
\end{aligned}
$$

The result follows from (3.1) and the Blaschke condition of order $\alpha-1 / 2$.

(ii) Introduce a new coordinate system $\left(z_{1}, z_{2}\right)$ in a neighborhood $V_{\zeta}$ of each $\zeta \in \partial D$ such that the $(1,0)$-forms $d z_{1}, d z_{2}$ constitute an orthogonal basis and $d z_{1}=$ $\lambda \partial \rho$, where $\lambda$ is a normalizing factor. Let $\Theta=\sum \Theta^{j k}$, where $\Theta^{j k}=\Theta_{j k} d z_{j} \wedge d \bar{z}_{k}$. Note that the decomposition of $\Theta$ does not depend on the choice of the basis $d z_{1}$, $d z_{2}$. Hence the decomposition is valid globally in a neighborhood of $\partial D$.

The inequalities in (i) and (ii) of Theorem 3.1 can be written in the equivalent forms respectively

$$
\int_{D}|\rho|^{\alpha-1 / 2}\left|\Theta_{22}\right| d V \lesssim B\left(\Theta, \alpha-\frac{1}{2}\right)
$$




$$
\int_{D}|\rho|^{\alpha}\left(\left|\Theta_{12}\right|+\left|\Theta_{21}\right|\right) d V \lesssim B\left(\Theta, \alpha-\frac{1}{2}\right)
$$

Since $\Theta=\bar{\Theta}$, we have $\Theta_{k j}=-\bar{\Theta}_{j k}$. Put $\omega=|\rho|^{\alpha / 2-1 / 4} d z_{1}+\theta|\rho|^{\alpha / 2+1 / 4} d z_{2}$, where $\theta$ is a complex valued function with $|\theta|=1$. Then

$$
\omega \wedge \bar{\omega}=|\rho|^{\alpha-1 / 2} d z_{1} \wedge d \bar{z}_{1}+|\rho|^{\alpha+1 / 2} d z_{2} \wedge d \bar{z}_{2}+|\rho|^{\alpha} \theta d z_{2} \wedge d \bar{z}_{1}+|\rho|^{\alpha} \bar{\theta} d z_{1} \wedge d \bar{z}_{2}
$$

and

$$
\begin{aligned}
\Theta \wedge \frac{\omega \wedge \bar{\omega}}{i}= & |\rho|^{\alpha-1 / 2} \Theta^{22} \wedge \frac{d z_{1} \wedge d \bar{z}_{1}}{i}+|\rho|^{\alpha+1 / 2} \Theta^{11} \wedge \frac{d z_{2} \wedge d \bar{z}_{2}}{i} \\
& +|\rho|^{\alpha} \theta \Theta^{12} \wedge \frac{d z_{2} \wedge d \bar{z}_{1}}{i}+|\rho|^{\alpha} \bar{\theta} \Theta^{21} \wedge \frac{d z_{1} \wedge d \bar{z}_{2}}{i}
\end{aligned}
$$

Take $\theta=\frac{1}{i} \exp \left(\frac{1}{i} \arg \Theta_{12}\right)$. Then

$$
\theta \Theta_{12}=\left|\Theta_{12}\right| \frac{1}{i} \text { and } \bar{\theta} \Theta_{21}=\left|\Theta_{21}\right| \frac{1}{i}
$$

Substituting this in (3.4), we get

$$
\begin{aligned}
\Theta \wedge \frac{\omega \wedge \bar{\omega}}{i}= & |\rho|^{\alpha-1 / 2} \Theta^{22} \frac{d z_{1} \wedge d \bar{z}_{1}}{i}+|\rho|^{\alpha+1 / 2} \Theta^{11} \wedge \frac{d z_{2} \wedge d \bar{z}_{2}}{i} \\
& -4|\rho|^{\alpha}\left|\Theta_{12}\right| d V-4|\rho|^{\alpha}\left|\Theta_{21}\right| d V .
\end{aligned}
$$

Since $\Theta \wedge \omega \wedge \bar{\omega} / i$ is a positive measure, we get

$$
\int_{D}|\rho|^{\alpha}\left(\left|\Theta_{12}\right|+\Theta_{21} \mid\right) d V \lesssim \int_{D}|\rho|^{\alpha+1 / 2}\left|\Theta_{11}\right| d V+\int_{D}|\rho|^{\alpha-1 / 2}\left|\Theta_{22}\right| d V .
$$

By (i) and the Blaschke condition of order $\alpha-1 / 2$, we get the result.

LeMmA 3.2. Let $g$ be a continuous function in $\bar{D}$ satisfying $g \equiv 0$ in some neighborhood of the origin. We define

$$
(K g)(z)=\int_{0}^{1} g(t z) d t, \quad z \in \bar{D} .
$$

(i) If $\alpha>0$, there is a constant $C_{\alpha}>0$ such that

$$
\int_{D}|\rho(z)|^{\alpha-1}|K g(z)| d V \leq C_{\alpha} \int_{D}|\rho(z)|^{\alpha}|g(z)| d V .
$$

(ii) There is a constant $C>0$ such that

$$
\int_{\partial D}|K g(z)| d \sigma \leq C \int_{D}|g(z)| d V .
$$


Proof. (i) See Lemma 3.1 in the chapter II of [Sko].

(ii) Let $t \cdot \partial D=\{w ; w=t z, z \in \partial D\}$. Then for some $c>0$,

$$
\begin{aligned}
\int_{\partial D}|K g(z)| d \sigma & =\int_{\partial D}\left|\int_{0}^{1} g(t z) d t\right| d \sigma \\
& \leq \int_{c}^{1} d t \int_{\partial D}|g(t z)| d \sigma(z), \quad \text { by Fubini's Theorem } \\
& \lesssim \int_{c}^{1} \frac{d t}{t^{3}} \int_{w \in t \cdot \partial D}|g(w)| d \sigma(w) \\
& \lesssim \int_{D}|g(z)| d V(z), \quad \text { by integrating by parts. }
\end{aligned}
$$

Theorem 3.3. Let $\alpha>0$. Let $\Theta$ be a d-closed, positive smooth $(1,1)$-form satisfying the Blaschke condition of order $\alpha-1 / 2$. Then there exists a real valued solution $v$ to $i \partial \bar{\partial} v=\Theta$ satisfying

$$
\int_{D}|\rho(z)|^{\alpha-1}|v(z)| d V \lesssim B\left(\Theta, \alpha-\frac{1}{2}\right) .
$$

Proof. Assuming without loss of generality that $D$ contains the origin, we apply the Poincaré-Cartan homotopy operator $P$ to $\Theta$. Then $d P \Theta=\Theta$. Note that if $\Theta=\sum \Theta_{j k} d z_{j} \wedge d \bar{z}_{k}$, then $P \Theta$ can be written by

$$
\begin{aligned}
P \Theta(z) & =\sum_{j=1}^{2}\left(\sum_{k=1}^{2} \int_{0}^{1} t z_{k} \Theta_{k j}(t z) d t\right) d \bar{z}_{j}-\sum_{k=1}^{2}\left(\sum_{j=1}^{2} \int_{0}^{1} t \bar{z}_{j} \Theta_{k j}(t z) d t\right) d z_{k} \\
& =f+\bar{f}=P^{\prime} \Theta+P^{\prime \prime} \Theta .
\end{aligned}
$$

From $\Theta=\bar{\Theta}$, we see $\Theta_{k j}=-\bar{\Theta}_{j k}$. Thus $\bar{\partial} f=0$. It suffices to show that

$$
\int_{D}|\rho|^{\alpha}\left(|f|+\frac{|\bar{\partial} \rho \wedge f|}{|\rho|}\right) d V \lesssim B\left(\Theta, \alpha-\frac{1}{2}\right) .
$$

Indeed if $f$ satisfies (3.6), then we can apply Theorem 2.1. Thus there exists a solution $u$ to $\bar{\partial} u=f$ such that

$$
\int_{D}|\rho(z)|^{\alpha-1}|u(z)| d V \lesssim B\left(\Theta, \alpha-\frac{1}{2}\right) .
$$


Then $v=2 \operatorname{Re} u$ is a real valued solution of the equation $i \partial \bar{\partial} v=\Theta$ satisfying the inequality (3.5). Now we will show (3.6). For sufficiently small $\eta>0$ choose a smooth cutoff function $\psi_{\eta}, 0 \leq \psi_{\eta} \leq 1$ satisfying

$$
\psi_{\eta}(z)= \begin{cases}1, & z \in\left\{z \in \mathbb{C}^{2} ;|\rho(z)| \leq \eta / 2\right\} \\ 0, & z \in\left\{z \in \mathbb{C}^{2} ;|\rho(z)| \geq \eta\right\}\end{cases}
$$

Decompose $\Theta=\left(1-\psi_{\eta}\right) \Theta+\psi_{\eta} \Theta$. Let $P^{\prime} \Theta(z)=f(z)$. Since $\psi_{\eta} \Theta_{1}$ has compact support in $D,(3.6)$ is clear. Hence we may assume that $\Theta \equiv 0$ in $D_{\eta}=\{z \in D$ : $\rho(z)<-\eta\}$. By (i) of Lemma 3.2, we obtain

$$
\begin{aligned}
\int_{D}|\rho(z)|^{\alpha}|f(z)| d V & \lesssim \int_{D}|\rho(z)|^{\alpha} \sum_{j, k}\left(\int_{0}^{1}\left|\Theta_{j k}(t z)\right| d t\right) d V \\
& \lesssim \int_{D}|\rho(z)|^{\alpha+1}|\Theta(z)|
\end{aligned}
$$

Since $|\bar{\partial} \rho \wedge f|=\left|\bar{\partial} \rho \wedge P^{\prime} \Theta\right| \lesssim\left|P^{\prime}\left(\Theta^{12}+\Theta^{22}\right)\right|$, (3.6) will follow from (3.7) and

$$
\int_{D}|\rho(z)|^{\alpha-1}\left|P^{\prime}\left(\Theta^{12}+\Theta^{22}\right)(z)\right| d V \lesssim B\left(\Theta, \alpha-\frac{1}{2}\right) d V
$$

Let $\left|\Theta^{2}(z)\right|=\left|\Theta^{12}(z)\right|+\left|\Theta^{22}(z)\right|$. Then by (i) of Lemma 3.2, (3.2) and (3.3), we obtain

$$
\begin{aligned}
\int_{D}|\rho(z)|^{\alpha-1}\left|P^{\prime}\left(\Theta^{12}+\Theta^{22}\right)(z)\right| d V & \lesssim \int_{D}|\rho(z)|^{\alpha-1}\left(\int_{0}^{1}\left|\Theta^{2}(t z)\right| d t\right) d V \\
& \lesssim \int_{D}|\rho(z)|^{\alpha}\left|\Theta^{2}(z)\right| d V \\
& \lesssim B\left(\Theta, \alpha-\frac{1}{2}\right) d V
\end{aligned}
$$

\section{The proof of Theorem 1.2}

We recall that

$$
D_{s}=\left\{\left(z_{1}, z_{2}\right) \in \mathbb{C}^{2} ; \rho\left(z_{1}, z_{2}\right)=\left|z_{1}\right|^{2}+c \cdot \exp \left(-\frac{1}{\left|z_{2}\right|^{s}}\right)-1<0\right\}
$$

where $0<s<1$ and $c=\exp (1+2 / s)$.

Let $\Theta$ be a $d$-closed, positive smooth (1,1)-form satisfying the Blaschke condition of order $-1 / 2$. By the same argument as in the proof of Theorem 1.1, it is 
enough to show that there exists a real valued function $v$ such that $i \partial \bar{\partial} v=\Theta$ in $D_{s}$ and

$$
\int_{\partial D_{s}}|v(z)| d \sigma(z) \lesssim B\left(\Theta,-\frac{1}{2}\right) .
$$

As in $\S 3$, the essential part of the proof of $(4.1)$ is the following $L^{1}\left(\partial D_{s}\right)$-estimates for $\bar{\partial}$.

Theorem 4.1. Let $f \in C_{(0,1)}^{\infty}\left(\bar{D}_{s}\right)$ and $\bar{\partial} f=0$ in $\bar{D}_{s}$. There is a solution $u \in C\left(\bar{D}_{s}\right)$ to $\bar{\partial} u=f$ in $D_{s}$ satisfying

$$
\int_{\partial D_{s}}|u(z)| d \sigma \leq C \int_{\partial D_{s}}|f(z) \wedge \bar{\partial} \rho(z)| d \sigma .
$$

Proof. Let $U$ be a small neighborhood of $\bar{D}_{s}$. Define

$$
\phi(\zeta, z)=\sum_{j=1}^{2} \frac{\partial \rho}{\partial \zeta_{j}}(\zeta)\left(\zeta_{j}-z_{j}\right), \quad(\zeta, z) \in U \times \mathbb{C}^{2}
$$

By Lemma 2 of [Ver], we see that there are constants $\eta, c>0$ depending only on $s$ such that for $M=1 / 32$, we have

$$
2 \operatorname{Re} \phi(\zeta, z) \geq \rho(\zeta)-\rho(z)+c e^{-1 / M|\zeta-z|^{2 s}}
$$

for $\zeta \in U,|\zeta-z|<\eta$.

Now we will construct a solution operator for the $\bar{\partial}$-equation. Because we need the $L^{1}\left(\partial D_{s}\right)$-estimate for $\bar{\partial}$, we use the well-known formula obtained from the Cauchy-Fantappiè kernel.

Let $W(\zeta, z)=\partial \rho(\zeta) / \phi(\zeta, z)$ and $\hat{W}(\zeta, z, \lambda)=\lambda W(\zeta, z)+(1-\lambda) B(\zeta, z)$, where $0 \leq \lambda \leq 1$ and $B(\zeta, z)=\partial_{\zeta}|\zeta-z|^{2} /|\zeta-z|^{2}$. The Cauchy-Fantappiè kernel $\Omega_{0}(\hat{W})$ of order 0 generated by $\hat{W}$ is defined by

$$
\Omega_{0}(\hat{W})=\frac{1}{(2 \pi i)^{2}} \hat{W} \wedge \bar{\partial}_{\zeta, \lambda} \hat{W}
$$

$\Omega_{0}(W)$ is defined in the same way, with $W$ instead of $\hat{W}$. For more details see Range [Ra1]. For $f \in C_{(0,1)}^{\infty}\left(\bar{D}_{s}\right)$, define

$$
\mathbb{B} f(z)=\int_{\zeta \in D_{s}} f(\zeta) \wedge \Omega_{0}(B), \quad z \in D_{s}
$$


and

$$
\mathbb{T}^{+} f(z)=\int_{\partial D_{s} \times[0,1]} f(\zeta) \wedge \Omega_{0}(\hat{W}), \quad z \in D_{s} .
$$

Then we obtain a solution $u(z)=\left(\mathbb{T}^{+}-\mathbb{B}\right) f(z)$ for the $\bar{\partial}$-equation.

Since $f \in C_{(0,1)}^{\infty}\left(\bar{D}_{s}\right), \mathbb{T}^{+} f$ and $\mathbb{B} f$ can be continuously extended up to the boundary of $D_{s}$ [Ra2]. In order to estimate the boundary values of $u=\left(\mathbb{T}^{+}-\mathbb{B}\right) f$ in terms of the boundary values of $f$, we have to express the integral $\mathbb{B} f$ in a different form using the dual form of $W(\zeta, z)$. Define $\phi^{*}(\zeta, z)=-\phi(z, \zeta)$ and $W^{*}(\zeta, z)=\partial_{\zeta}(z) / \phi^{*}(\zeta, z)$. Note that $\phi^{*}$ is well-defined for $(\zeta, z) \in \bar{D}_{s} \times\left(U \backslash D_{s}\right)$. We define

$$
\mathbb{T}^{-} f(z)=\int_{\partial D_{s} \times[0,1]} f(\zeta) \wedge \Omega_{0}\left(\hat{W}^{*}\right), \quad z \in U \backslash D_{s}
$$

We see that if $z \in \partial D_{s}$, then the solution $u(z)=\mathbb{T}^{+} f(z)-\mathbb{T}^{-} f(z)$ [Sha]. Integrating over $\lambda$, we have for some constants $a, a^{*}$,

$$
\begin{aligned}
& \mathbb{T}^{+} f(z)=\frac{a}{(2 \pi i)^{2}} \int_{\zeta \in \partial D_{s}} f(z) \wedge E(\zeta, z), \\
& \mathbb{T}^{-} f(z)=\frac{a^{*}}{(2 \pi i)^{2}} \int_{\zeta \in \partial D_{s}} f(z) \wedge E^{*}(\zeta, z),
\end{aligned}
$$

where

$$
E(\zeta, z)=\frac{\partial_{\zeta} \rho(\zeta) \wedge \partial_{\zeta}|\zeta-z|^{2}}{\phi(\zeta, z)|\zeta-z|^{2}}, \quad E^{*}(\zeta, z)=\frac{\partial_{\zeta} \rho(z) \wedge \partial_{\zeta}|\zeta-z|^{2}}{\phi^{*}(\zeta, z)|\zeta-z|^{2}}
$$

Let $f=f_{1}+f_{2}$, where $f_{1}$ is the normal component and $f_{2}$ the tangential component of $f$. Then $f_{1}=g \bar{\partial} \rho$ for some function $g \in C^{\infty}\left(\partial D_{s}\right)$. Thus we have

$$
\begin{aligned}
\mathbb{T}^{+} f_{1}(z) & =a \int_{\partial D_{s}} f_{1} \wedge E \\
& =a \int_{\partial D_{s}} g \bar{\partial} \rho \wedge E \\
& =a \int_{\partial D_{s}} g d \rho \wedge E \\
& =0, \quad \text { by Stokes' Theorem. }
\end{aligned}
$$

Thus we have $\mathbb{T}^{+} f(z)=\mathbb{T}^{+} f_{2}(z)$ on $\partial D_{s}$. Similarly $\mathbb{T}^{-} f(z)=\mathbb{T}^{-} f_{2}(z)$ on $\partial D_{s}$. Therefore we obtain

$$
u(z)=\mathbb{T}^{+} f_{2}(z)-\mathbb{T}^{-} f_{2}(z) \text { for } z \in \partial D_{s} .
$$


The inequality (4.2) can be written in the equivalent form

$$
\int_{\partial D_{s}}|u(z)| d \sigma(z) \lesssim \int_{\partial D_{s}}\left|f_{2}(z)\right| d \sigma(z) .
$$

To prove the inequality (4.4), by the Fubini theorem, it suffices to show that the following integrals,

$$
\int_{\partial D_{s}}|E(\zeta, z)| d V(z), \quad \int_{\partial D_{s}}\left|E^{*}(\zeta, z)\right| d V(z)
$$

are uniformly bounded for each $\zeta \in \partial D_{s}$. For the first integral of (4.5), we use the coordinate (2.2) and the inequality (4.3). By the compactness of $\partial D_{s}$ and the smoothness of the kernel $E$ on $\bar{D}_{s} \times \bar{D}_{s} \backslash\left\{(\zeta, \zeta): \zeta \in \partial D_{s}\right\}$, it suffices to show that for each $\zeta \in \partial D_{s}$ and sufficiently small ball $B_{\eta}(\zeta)$,

$$
\begin{aligned}
\int_{\partial D_{s} \cap B_{\eta}(\zeta)}|E(\zeta, z)| d \sigma(z) & \lesssim \int_{\partial D_{s} \cap B_{\eta}(\zeta)} \frac{d \sigma(z)}{|\zeta-z||\phi(\zeta, z)|} \\
& \lesssim \int_{t=\left(t_{2}, t^{\prime \prime}\right)} \frac{d t_{2} d t^{\prime \prime}}{\left|t^{\prime \prime}\right|\left(\left|t_{2}\right|+e^{\left.-M /\left|t^{\prime \prime}\right|^{s}\right)}\right.} \\
& \lesssim \int_{\left|t^{\prime \prime}\right|<1, t^{\prime \prime} \in \mathbb{R}^{2}} \frac{d t^{\prime \prime}}{\left|t^{\prime \prime}\right|\left|t^{\prime \prime}\right|^{s}} \\
& \lesssim \int_{0}^{1} \frac{d r}{r^{s}} \lesssim 1 .
\end{aligned}
$$

On the other hand, since $\phi^{*}(\zeta, z)=-\phi(z, \zeta)$, it follows that

$$
2 \operatorname{Re} \phi^{*}(\zeta, z) \leq-\rho(\zeta)+\rho(z)-c e^{-M /|\zeta-z|^{s}}
$$

for $\zeta \in \bar{D}_{s},|\zeta-z|<\eta$. Hence the second integral of (4.5) can be estimated as the first one.

Now we return to the proof of (4.1). Let $d P \Theta=\Theta, P \Theta=f+\bar{f}$ and $P^{\prime} \Theta=f$ as in the proof of Theorem 3.3. Then it is enough to show that

$$
\int_{\partial D_{s}}\left|f_{2}(z)\right| d \sigma(z) \lesssim B\left(\Theta,-\frac{1}{2}\right)
$$

Note that $\left|f_{2}\right| \lesssim\left|P^{\prime} \Theta^{2}\right|$. By (ii) of Lemma 3.2, (3.2) and (3.3), we have

$$
\begin{aligned}
\int_{\partial D_{s}}\left|P^{\prime}\left(\Theta^{2}\right)(z)\right| d \sigma(z) & \lesssim \int_{\partial D_{s}} \int_{0}^{1}\left|\Theta^{2}(t z)\right| d t d \sigma(z) \\
& \lesssim \int_{D_{s}}\left|\Theta^{2}\right| d V \\
& \lesssim B\left(\Theta,-\frac{1}{2}\right) .
\end{aligned}
$$




\section{References}

[And] M. Andersson, Solution formulas for the $\partial \bar{\partial}$-equation and weighted Nevanlinna classes in the polydisc, Bull. Sci. Math., 109 (1985), 135-154.

[BeAn] B. Berndtsson and M. Andersson, Henkin-Ramirez formulas with weight factors, Ann. Inst. Fourier, 32 (1982), 91-110.

[BoCh] A. Bonami and P. Charpentier, Solutions de l'équation $\bar{\partial}$ et zéros de la classe Nevanlinna dans certains domaines faiblement pseudo-convexes, Ann. Inst. Fourier, 32 (1982), 53 89.

[BCD] J. Bruna, P. Charpentier and Y. Dupain, Zero varieties for the Nevanlinna class in convex domains of finite type in $\mathbb{C}^{n}$, Ann. of Math., 147 (1998), 391-415.

[CNS] D.C. Chang, A. Nagel and E.M. Stein, Estimates for the $\bar{\partial}$-Neumann problem for pseudoconvex domains in $\mathbb{C}^{2}$ of finite type, Proc. Nat. Acad. Sci. USA, 85 (1988), 8771-8774.

[Cha] P. Charpentier, Caractérisations des zéros des fonctions de certaines classes de type Nevanlinna dans le bidisque, Ann. Inst. Fourier, 34 (1984), 57-98.

[Che] S.C. Chen, Real analytic boundary regularity of the Cauchy kernel on convex domains, Proc. Amer. Math. Soc., 108-2 (1990), 423-432.

[Cum] A. Cumenge, Zero sets of functions in the Nevanlinna or the Nevanlinna-Djrbachian classes, Pacific J. Math., 199 (2001), 79-92.

[DaHe] A. Dautov and G.M. Henkin, Zeros of holomorphic functions of finite order and weighted estimates for solutions of the $\bar{\partial}$-equation, Math. USSR-Sb., 35 (1979), 449-459.

[DiMa] K. Diederich and E. Mazzilli, Zero varieties for the Nevanlinna class on all convex domains of finite type, to appear in Nagoya J. Math.

[Hen] G.M. Henkin, H. Lewy's equation and analysis on a pseudoconvex manifold, II., Math. USSR-Sb., 31 (1977), 63-94.

[Le1] P. Lelong, Fonctionelles analytiques et fonctions entières ( $n$ variables), Montreal: Les presses de l'université de Montreal (1968).

[Le2] P. Lelong, Fonctions plurisousharmoniques et formes differentielles positive, London: Gordon and Breach (1968).

[Pol] J.C. Polking, The Cauchy-Riemann equations in convex domains, Proc. Symp. Pure Math., 52 (1991), 309-322.

[Ra1] R.M. Range, Holomorphic Functions and Integral Representations in Several Complex Variables, Springer-Verlag, New York, 1986.

[Ra2] R.M. Range, On Hölder and BMO estimates for $\bar{\partial}$ on convex domains in $\mathbb{C}^{2}$, The Journal of Geometric Analysis, 2-6 (1992), 575-584.

[Ra3] R.M. Range, On $\bar{\partial}$-problems on (pseudo)-convex domains, Topics in complex analysis Banach center publications, 31 (1995), Institute of Mathematics, Polish Academy of Sciences, Warsawa.

[Sha] M.-C. Shaw, Prescribing zeros of functions in the Nevanlinna class on weakly pseudoconvex domains in $\mathbb{C}^{2}$, Trans. Amer. Math. Soc., 313 (1989), 407-418.

[Sko] H. Skoda, Valeurs au bord pour les solutions de l'opérateur $d^{\prime \prime}$, et caractérisation des zéros des fonctions de la classe de Nevanlinna, Bull. Soc. Math. France, 104 (1976), 225-299.

[Ver] J. Verdera, $L^{\infty}$-continuity of Henkin operators solving $\bar{\partial}$ in certain weakly pseudoconvex domains of $\mathbb{C}^{2}$, Proc. Roy. Soc. Edinburgh, 99 (1984), 25-33. 
Heungue Ahn Department of Mathematics SeOUl Natoinal University Seoul 151-742, South Korea

E-mail: hjahn@math.snu.ac.kr

Hong Rae Cho

Department of Mathematics Education Andong National University Andong 760-749, South Korea E-mail: chohr@andong.ac.kr 